\title{
EVOLUTIONS OF SENTINEL-2 LEVEL-2A CLOUD MASKING ALGORITHM: SEN2COR PROTOTYPE FIRST RESULTS
}

\author{
Jérôme Louis ${ }^{(1)}$, Bringfried Pflug ${ }^{(2)}$, Vincent Debaecker ${ }^{(1)}$, Uwe Mueller-Wilm $^{(3)}$, \\ Rosario Quirino Iannone ${ }^{(4)}$, Valentina Boccia ${ }^{(5)}$ Ferran Gascon $^{(5)}$ \\ (1) Telespazio France, 26 Av. JF Champollion, 31023 Toulouse Cedex 1 (France), \\ email: jerome.louis@telespazio.com, vincent.debaecker@telespazio.com, \\ (2) German Aerospace Centre, Remote Sensing Technology Institute, email: bringfried.pflug@dlr.de \\ (3) TPZV-D - Telespazio Vega Deutschland - A Leonardo / Thales Company \\ email: uwe.mueller-wilm@ telespazio-vega.de \\ (4) RHEA SpA, email: rosario.quirino.iannone@esa.int \\ (5) European Space Agency (ESA), European Space Research Institute (ESRIN), Italy \\ email: valentina.boccia@esa.int, ferran.gascon@esa.int
}

\begin{abstract}
Reliable cloud screening remains a critical issue for the analysis of optical imagery. In this work we present the recent evolutions of the Cloud Screening and Scene Classification module of Sen2Cor processor for the Copernicus Sentinel-2 mission. In addition to a Level-2A surface reflectance product, Sen2Cor provides a Scene Classification (SCL) map divided into 11 classes. The information provided by this map is of great interest for automated processing chains. It can be used to mask out clouds, cloud shadow, water, snow/ice from the Sentinel-2 imagery, so that downstream processing can be performed on clear land pixels, suitable for time-series analysis and quantitative remote sensing.

The performance and limitations of the current algorithm are recalled. The updates aimed at improving the overall accuracy of the cloud screening are described (efficient topographic shadows computation, mitigation of false snow detection in clouds and mitigation of false clouds detection on bright targets). Preliminary results based on a Sen2Cor prototype are discussed.
\end{abstract}

Index Terms - Cloud screening, Classification, Copernicus, Sentinel-2, Sen2Cor, Validation, Cloud mask

\section{INTRODUCTION}

The Sentinel-2 mission [1] is dedicated to land and coastal waters monitoring at high spatial resolution in the optical domain. Sentinel-2 serves a large range of applications and services such as precision agriculture, water quality monitoring, forests management and natural disasters mapping.
ESA has been using Sen2Cor [2] for the systematic production of Sentinel-2 Level-2A data over the World since December 2018 (over Europe since June 2017).

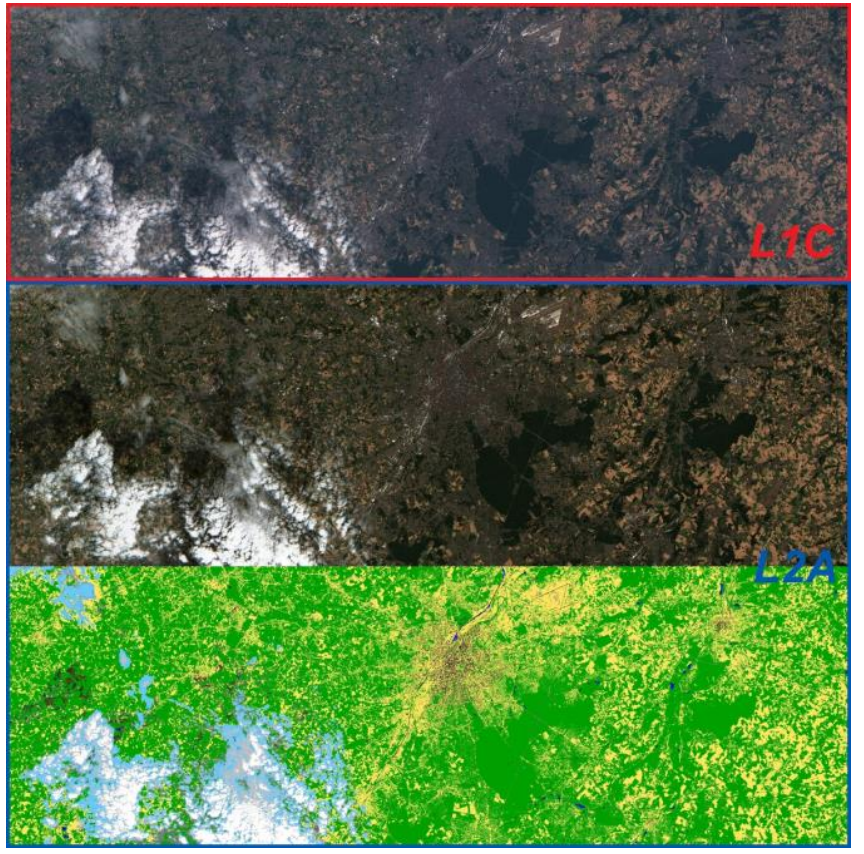

Figure 1: Bruxelles region, Belgium, Sentinel-2 image acquired on $8^{\text {th }}$ of August 2020. From top to bottom: (1) Level-1C TOA reflectance input image, (2) Level-2A surface reflectance image, (3) Level-2A scene classification product.

An accurate cloud screening of satellite observations is a precondition for the development and delivery of highquality applications and added-value products. Despite the scene classification map provided by Sen2Cor does not constitute a land cover classification map in a strict sense its initial purpose being internal usage in Sen2Cor's atmospheric correction module to distinguish between 
cloudy -, clear - and water pixels -, it has proven to be useful for users to identify valid pixels for their applications.

The evolutions described in this paper aimed at improving its overall accuracy in terms of cloud screening and should have a positive impact on atmospheric correction to derive accurate surface reflectance.

It is interesting to note that several comparison activities of different cloud masking processors available have been performed, either by individual or by agencies [3], [4], [5]. In 2019, ESA and NASA jointly organized the first Cloud Masking Inter-comparison Exercise (CMIX) initiative [6] for cloud masking as they did with ACIX [7] for atmospheric correction. CMIX results should be available by the time of IGARSS 2021.

Cloud masking will remain a hot topic, as a larger amount of automated applications will emerge in the coming years with the on-line availability of satellite imagery data on platforms like Copernicus Data \& Information Access Services (DIAS) [8].

\section{OFFICIAL CLOUD SCREENING AND SCENE CLASSIFICATION ACCURACY}

Classification accuracy is evaluated within Sentinel-2 Mission Performance Centre (S2 MPC) by comparison of the Sen2Cor outputs with reference samples. The reference samples are generated by visual inspection and labelling of a validation data set, which was determined by stratified random sampling.

Current analysis of classification accuracy for Sen2Cor 2.5 (same algorithm as v.2.8) using auxiliary information from ESA Land Cover Climate Change Initiative (CCI) project [9] is based on 14 Sentinel-2 L2A images over 13 test sites. Validation data set represents different atmospheric conditions (e.g. cloud cover), latitudes (various solar angles and seasons), topography (flat, rough and mountainous terrain), and land cover types (agricultural area, forests, water bodies, arid area, urban area, deserts, permanent ice, and active volcanos).

The results of the complete accuracy assessment per test site is presented in L2A Data Quality Report [10]. Overall Accuracy (OA) of clear pixels aggregates results for the Sen2Cor classes "vegetation", "non-vegetated" and "water". OA of clouds aggregates results for Sen2Cor classes "cloud medium probability", "cloud high probability" and "thin cirrus". The recognition of clear pixels reached an average OA of $91.5 \%$ and the recognition of clouds reached an OA of $94.8 \%$.

The current known limitations of the algorithm are some false cloud detections in bright areas like arid regions or cities, false snow detections in high altitude clouds, as well as limited cloud shadow accuracy for complex atmospheric conditions. There is still a need to improve the accuracy of the cloud screening and the section below describes the approach taken to reach that goal.

\section{CLOUD SCREENING AND SCENE CLASSIFICATION UPDATES}

The official scene classification algorithm is described in details in the latest L2A ATBD version 2.9 [11]. Motivated by the users' feedback and positive emulation of the CMIX initiative, several evolutions have been prepared regarding the future version of Scene Classification (expected in Sen2Cor v2.10):

- Improvement of topography/casted shadows + acceleration of casting shadow detection with cython implementation

- Update of snow climatology to reduce false snow detection

- MSI Parallax characteristics for cloud screening using cloud top height estimation (cloud shadow algorithm, false snow and bright pixels handling)

- Inclusion of cloud / cloud shadow / snow dilation

\subsection{Topographic shadows}

The accuracy of the topographic shadows classification shall improve in the near future with the more precise Copernicus digital elevation model (DEM) [12]. In addition, a ray tracing casted shadow algorithm based on [13] has been developed in Sen2Cor prototype to improve the detection of topographic shadows with low solar angles. It should replace the current gdaldem hillshade algorithm in future versions. Thanks to a cython implementation of this algorithm, the processing time has been drastically reduced and it is now possible to perform the topographic shadows detection at $20 \mathrm{~m}$ resolution within few seconds.

\subsection{Mitigation of false snow detection clouds}

We noticed that false snow detections in clouds could perturb the adjacency correction, leading to an overcorrection in tiles almost covered by clouds. It is therefore important to mitigate the false snow detection in high altitude clouds.

Several methods have been tested. In the end, we selected an effective method for a global mission like Sentinel-2 by relying - not exclusively - on an updated snow climatology based on 12 monthly Snow condition images, derived from a post-processing of the 52 Snow Conditions images from ESA CCI data package [14]. This postprocessing was found necessary, as native SNow Condition (SNC) weekly files were not able to catch the possibility of rare snow events or snow/ice on inland waters or icebergs.

Preliminary results show a noticeable improvement; nevertheless, extremely rare snow events like the ones occurred in January 2021 in Madrid or in Morocco could still be missed following that approach. A complementary algorithm based on instrumental parallax is therefore proposed in the next section. 


\subsection{MSI Parallax characteristics for cloud screening}

In push-broom instruments like Sentinel-2 MSI, a parallax exists between odd and even detectors as well as a parallax between bands [15]. Different methods, like [16], can be used to exploit this instrumental feature. We have tested a new method based on a novel Telespazio algorithm using OpenCV library. This method is tested to improve the cloud shadow algorithm by evaluating the cloud top height and restricting the area for cloud shadow determination. The algorithm of cloud top height estimation using Sentinel-2 MSI instrumental parallax is summarized hereafter:

- Useful S2 bands: Band B08 (resampled at $20 \mathrm{~m}$ ) and B8A (20 m)

- Pixel displacement is computed for the whole image (novel Telespazio algorithm using OpenCV)

- Pixel displacement is converted in cloud top height estimation (m) using B08/B8A parallax information from instrument characteristics and pixel resolution Remark: cloud top height is altitude above DEM. It is not altitude above sea level

- Statistics on the cloud height distribution are used to identify the regions of potential cloud shadow in the image (using sun angles).

- This information is then "crossed check" with potential cloud shadow pixel based on pixel radiometry (as in Sen2Cor v2.8)

- Clouds with cloud top height below $250 \mathrm{~m}$ are discarded from cloud mask for cloud shadow

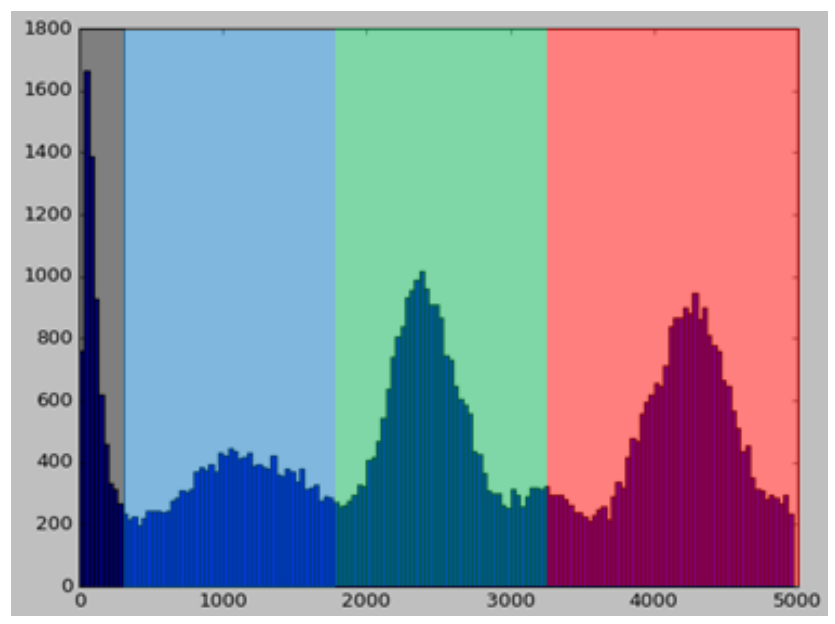

Figure 3: Histogram of pixel cloud height

Figure 3 shows the histogram of the computed cloud top height displayed in Figure 4. The cloud top height information is used also to mitigate the false snow detection in clouds and the false clouds detection on ground (e.g. natural bright targets or artificial bright targets).
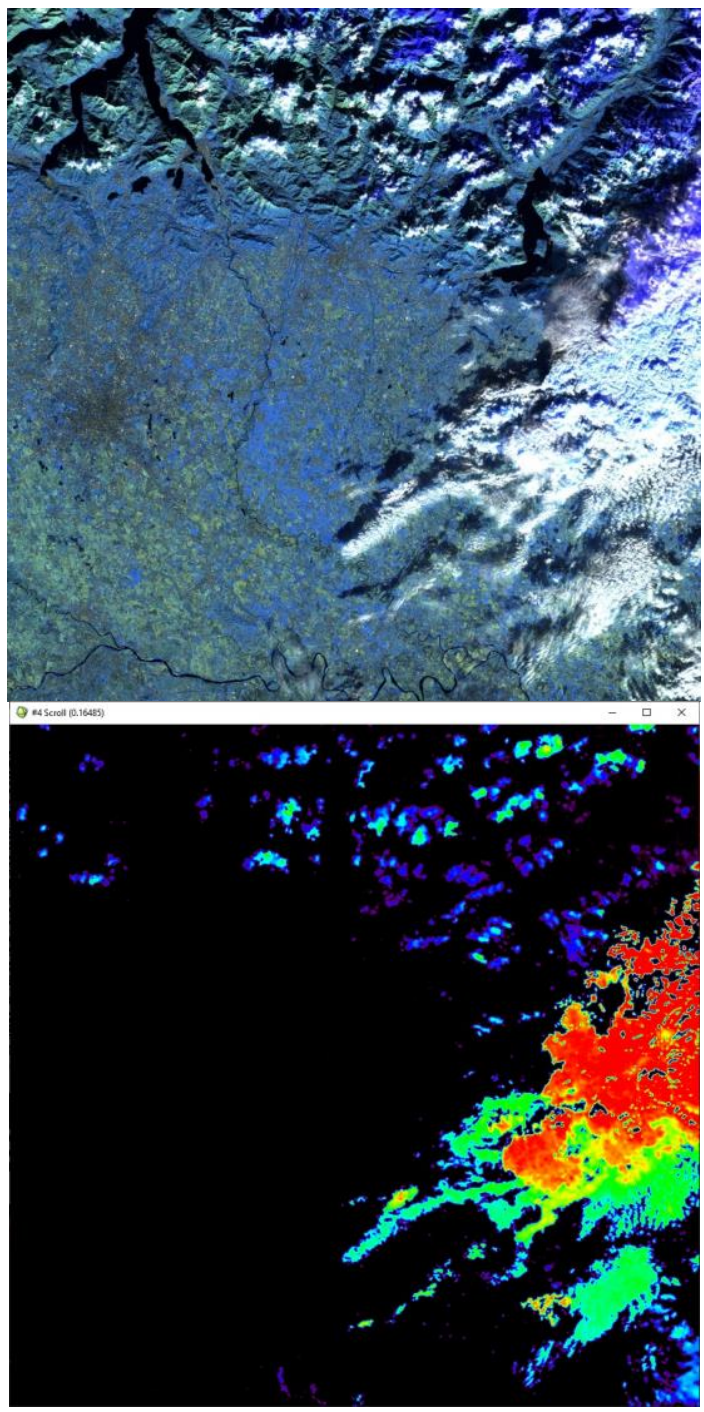

Figure 4: Top: False colour RGB color composite (R=B12, G=B11, $\mathrm{B}=\mathrm{B} 8 \mathrm{~A}$ ) acquired on $8^{\text {th }}$ November 2016 over Milan region, North of Italy Bottom: Visualization of computed top cloud height using MSI parallax

\subsection{Dilation of cloud, cloud shadow, snow masks}

A dilation of clouds, cloud shadows and snow masks is now available with the following characteristics:

- +80 m cloud mask

- +40 m cloud shadow mask

- +20 m snow mask

The dilation is performed carefully to avoid false cloud dilation leading to higher commission error:

- No cloud dilation in shoreline regions (bright sand / white caps issues)

- Only large clouds (>1000 pixels @ 20 m) are dilated above urban areas

- No cloud dilation in soil/snow boundary regions (mixed pixel spectral issue) 
- Only cloud with cloud probability > 65\% are dilated

- Very small clouds ( $<=3$ pixels $)$ are not dilated

\section{PRELIMINARY RESULTS \& DISCUSSION}

The prototype described in this abstract has been used to reprocess a set of 145 images from the CMIX datasets and the results should be available by the time of IGARSS 2021 and be further discussed. From preliminary results, it appears that the cloud borders are now much better identified as clouds without degrading the overall accuracy of the classification. Topographic shadows are better classified especially for low solar angles, thanks to the Copernicus DEM with improved resolution and the raytracing algorithm. The MSI parallax method to derive cloud top height looks very promising to address three main challenges: the cloud shadow identification, the mitigation of false snow detection in clouds and the mitigation of false cloud detection on bright targets. For some rare cases, we expect parallax computation issues related to very low altitude clouds or hazy conditions that could be addressed in the future relying on meteorological parameters like humidity, temperature and wind speed.

\section{CONCLUSION}

The performance of the Level-2A cloud screening and scene classification product generated by Sentinel-2 ground segment (S2 PDGS) using Sen2Cor is regularly assessed within Sentinel-2 Mission Performance Centre and by independent teams. In this abstract, we described the different methods proposed to reach a higher level of overall accuracy. Consolidated validation results will be presented at the IGARSS 2021 conference.

\section{ACKNOWLEDGMENTS}

We would like to thank the European Space Agency for funding this research activities.

\section{REFERENCES}

[1] Drusch, M.; Del Bello, U.; Carlier, S.; et al. (2012). "Sentinel2: ESA's Optical High-Resolution Mission for GMES Operational Services", Remote Sensing of Environment, 120, 25-36

[2] Richter, R.; Louis, J.; and Müller-Wilm, U. (2012). „Sentinel-2 MSI-Level 2A Products Algorithm Theoretical Basis Document", S2PAD-ATBD-0001, Issue 2.0, Telespazio VEGA Deutschland $\mathrm{GmbH}$, Darmstadt, Germany

[3] Zekoll, V.; Main-Knorn, M.; Louis, J.; Frantz, D.; Richter, R.; Pflug,B. "Comparison of Masking Algorithms for Sentinel-2 Imagery." Remote Sens. 2021, 13, 137. https://doi.org/10.3390/rs13010137
[4] Baetens, Louis; Desjardins, Camille; Hagolle, Olivier. 2019. "Validation of Copernicus Sentinel-2 Cloud Masks Obtained from MAJA, Sen2Cor, and FMask Processors Using Reference Cloud Masks Generated with a Supervised Active Learning Procedure" Remote Sens. 11, no. 4: 433. https://doi.org/10.3390/rs11040433

[5] Raiyani, Kashyap; Gonçalves, Teresa; Rato, Luís; Salgueiro, Pedro; Marques da Silva, José R. 2021. "Sentinel-2 Image Scene Classification: A Comparison between Sen2Cor and a Machine Learning Approach" Remote Sens. 13, no. 2: 300. https://doi.org/10.3390/rs13020300

[6] Cloud Masking Inter-comparison Exercise (CMIX) initiative: https://earth.esa.int/web/sppa/meetings-workshops/hosted-and-cosponsored-meetings/acix-ii-cmix-2nd-ws, accessed on 20/01/2021.

[7] G. Doxani et al. (2018), "Atmospheric Correction InterComparison Exercise", Remote Sensing, 10 (352), pp 1-18. DOI: doi:10.3390/rs10020352 ISSN 2072-4292

[8] Copernicus Data and Information Access Services (DIAS) https://www.copernicus.eu/en/access-data/dias accessed on $21 / 01 / 2021$

[9] Land Cover CCI Climate Research Data Package (CRDP) https://maps.elie.ucl.ac.be/CCI/viewer/download.php Accessed on $\underline{07 / 01 / 2019}$

[10] Sentinel-2 L2A Data Quality Report Issue 33 (January 2021) https://sentinels.copernicus.eu/documents/247904/685211/Sentinel -2-L2A-Data-Quality-Report-January-2021.pdf accessed on $21 / 01 / 2021$

[11] Level-2A Algorithm Theoretical Basis Document (ATBD) version $\quad 2.9 \quad$ https://sentinel.esa.int/web/sentinel/userguides/sentinel-2-msi/document-library accessed on 21/01/2021

[12] Copernicus Digital Elevation Model Product Handbook https://spacedata.copernicus.eu/web/cscda/datasetdetails?articleId $=394198$ accessed on $21 / 01 / 2021$

[13] Javier G. Corripio (2003) "Vectorial algebra algorithms for calculating terrain parameters from DEMs and solar radiation modelling in mountainous terrain", Int. J. Geographical Information Science, 2003, vol.17, no 1, 1-23.'

[14] Land Cover CCI Climate Research Data Package (CRDP) https://maps.elie.ucl.ac.be/CCI/viewer/download.php Accessed on $07 / 01 / 2019$.

[15] Gascon F., Bouzinac, C., Thépaut, O., et al. (2017). Copernicus Sentinel-2A Calibration and Products Validation Status, Remote Sens. 2017, 9(6), 584; doi:10.3390/rs9060584

[16] David Frantz, Erik Haß, Andreas Uhl, Johannes Stoffels, Joachim Hill, "Improvement of the Fmask algorithm for Sentinel-2 images: Separating clouds from bright surfaces based on parallax effects," Remote Sensing of Environment, Volume 215, 2018, Pages 471-481, https://doi.org/10.1016/j.rse.2018.04.046. 